\title{
Measuring prefrontal cortical activity during dual task walking in patients with Parkinson's disease: feasibility of using a new portable fNIRS device
}

Freek Nieuwhof ${ }^{1,2}$, Miriam F. Reelick ${ }^{1,2}$, Inbal Maidan ${ }^{3}$, Anat Mirelman ${ }^{3,4}$, Jeffrey M. Hausdorff ${ }^{3,4}$, Marcel G.M. Olde Rikkert ${ }^{1,2}$, Bastiaan R. Bloem ${ }^{1,2}$, Makii Muthalib ${ }^{5}$ and Jurgen A.H.R. Claassen ${ }^{1,2^{*}}$

\begin{abstract}
Background: Many patients with Parkinson's disease (PD) have difficulties in performing a second task during walking (i.e., dual task walking). Functional near-infrared spectroscopy (fNIRS) is a promising approach to study the presumed contribution of dysfunction within the prefrontal cortex (PFC) to such difficulties. In this pilot study, we examined the feasibility of using a new portable and wireless fNIRS device to measure PFC activity during different dual task walking protocols in PD. Specifically, we tested whether PD patients were able to perform the protocol and whether we were able to measure the typical fNIRS signal of neuronal activity.

Methods: We included 14 PD patients (age 71.2 \pm 5.4 years, Hoehn and Yahr stage II/III). The protocol consisted of five repetitions of three conditions: walking while (i) counting forwards, (ii) serially subtracting, and (iii) reciting digit spans. Ability to complete this protocol, perceived exertion, burden of the fNIRS devices, and concentrations of oxygenated $\left(\mathrm{O}_{2} \mathrm{Hb}\right)$ and deoxygenated $(\mathrm{HHb})$ hemoglobin from the left and right PFC were measured.

Results: Two participants were unable to complete the protocol due to fatigue and mobility safety concerns. The remaining 12 participants experienced no burden from the two fNIRS devices and completed the protocol with ease. Bilateral PFC $\mathrm{O}_{2} \mathrm{Hb}$ concentrations increased during walking while serially subtracting (left PFC $0.46 \mu \mathrm{mol} / \mathrm{L}$, $95 \%$ confidence interval $(\mathrm{Cl}) 0.12-0.81$, right PFC $0.49 \mu \mathrm{mol} / \mathrm{L}, 95 \% \mathrm{Cl} 0.14-0.84$ ) and reciting digit spans (left PFC $0.36 \mu \mathrm{mol} / \mathrm{L}, 95 \% \mathrm{Cl} 0.03-0.70$, right PFC $0.44 \mu \mathrm{mol} / \mathrm{L}, 95 \% \mathrm{Cl} 0.09-0.78)$ when compared to rest. HHb concentrations did not differ between the walking tasks and rest.

Conclusions: These findings suggest that a new wireless fNIRS device is a feasible measure of PFC activity in PD during dual task walking. Future studies should reduce the level of noise and inter-individual variability to enable measuring differences in PFC activity between different dual walking conditions and across health states.
\end{abstract}

Keywords: Spectroscopy, Near-infrared, Gait, Parkinson's disease, Prefrontal cortex, Hemodynamics

\footnotetext{
* Correspondence: jurgen.claassen@radboudumc.nl

${ }^{1}$ Donders Institute for Brain, Cognition and Behaviour, Nijmegen, The

Netherlands

2Departments of Neurology, Geriatric Medicine, and Radboud Alzheimer

Center, Radboud University Medical Center, Nijmegen, The Netherlands

Full list of author information is available at the end of the article
} 


\section{Background}

Many patients with Parkinson's disease (PD) have difficulties to perform a second task while walking (i.e., dual task walking), such as walking and talking or walking while paying attention to passing traffic [1]. As a consequence of performing two tasks at the same time, gait and/or their performance on the secondary task at hand deteriorates [2-4]. These difficulties in dual task walking often lead to increased disability, increased fall risk, and reduced quality of life $[1,5,6]$.

Mechanisms underlying difficulties in dual task walking are largely unclear. However, the prefrontal cortex (PFC), which is involved in human balance and locomotion [7], likely plays an important role. Although cognitive functions depending on the PFC are often affected in PD [8-10], patients may rely more on the PFC due to reduced movement automaticity of dysfunctional basal ganglia circuits $[1,11-13]$. Therefore, altered functioning of the PFC during dual task walking in PD might explain their difficulties and should therefore be further examined.

Functional near-infrared spectroscopy (fNIRS) is a promising method for measuring PFC activity during dual task walking [14-16]. With fNIRS, relative concentrations of oxygenated $\left(\mathrm{O}_{2} \mathrm{Hb}\right)$ and deoxygenated $(\mathrm{HHb})$ hemoglobin can be measured $[17,18]$. In typical neural activity as measured with fNIRS, increases in $\mathrm{O}_{2} \mathrm{Hb}$ and stable or slight decreases in $\mathrm{HHb}$ are present $[14,16,18,19]$. The use of fNIRS offers several advantages over other neuroimaging techniques. Compared to functional magnetic resonance imaging (fMRI), which has a higher spatial resolution and can reach subcortical areas, fNIRS is lightweight, easy to use, low cost, and can be portable [11, 14, 18]. Compared to electroencephalography (EEG), which can also be used during actual walking [20], fNIRS can provide higher spatial resolution, is easier to use, and more robust to head movement $[14,18,21]$. These advantages make fNIRS particularly attractive to be used for measurement during actual walking [11].

In healthy young and elderly persons, fNIRS was used to detect increased PFC activity during walking while talking [22, 23], while counting [24], and while serially subtracting [24-26]. Compared to these populations, PD patients generally are physically less fit. This might limit their ability to perform multiple task repetitions, which are needed for reliable fNIRS measurements. In addition, PD patients often show altered movement patterns such as restricted gait, dyskinesia, or bradykinesia. This might influence head position and head movement during walking and thereby further restrict the feasibility of using fNIRS to measure PFC activity during dual task walking.

Therefore, the primary aim of this pilot study was to examine the feasibility of measuring bilateral PFC activity in patients with PD during different dual task walking conditions using two lightweight, wireless fNIRS devices (Portalite fNIRS system). This was done in the context of the V-TIME project $[27,28]$ in which we applied fNIRS to study the role of the PFC in complex walking in PD patients. The first results of this project, which were recently published [13], focus on potential mechanisms underlying dual task difficulties in PD. Although outside the scope of this recently published work, details of pilot work concerning methodology and feasibility of using fNIRS in PD are crucial for the development of new studies to further disentangle the neural mechanisms underlying dual tasking in PD.

As a start for the development of appropriate protocols for such studies, this feasibility study incorporated several specific goals. First, we assessed feasibility by testing whether PD patients were able to perform several repetitions of dual task walking while wearing the devices and by registering their experience and perceived exertion. Second, we aimed to investigate whether we were able to record the expected typical fNIRS signal of neuronal activity in the PFC (the neuronal hemodynamic response [18]) as a consequence of dual task walking when compared to rest. We tested this at a group level, and at individual level, to explore potential inter-individual variability. Finally, we explored the sensitivity of our method to detect differences in $\mathrm{O}_{2} \mathrm{Hb}$ and $\mathrm{HHb}$ concentrations between dual task walking and rest and between dual tasks.

\section{Methods \\ Participants}

We recruited PD patients $(N=14)$ from the outpatient clinic of the Neurology Department of the Radboud University Medical Center in the Netherlands. This number of 14 participants was based on similar studies in other populations, in which 6 [29], 11 [22], and 17 [25] participants were sufficient to measure PFC activity during walking tasks. Inclusion criteria were (1) age 60-85 years, (2) clinical diagnosis of idiopathic Parkinson's Disease, according to the UK Brain Bank criteria, (3) Hoehn and Yahr stage II to III (while on medication), (4) an increased risk of falling as indicated by the treating physician, or reflected by actual falling incidents within 6 months prior to the study, and (5) able to walk at least $5 \mathrm{~min}$ without help (walking aids were allowed). Exclusion criteria were (1) psychiatric co-morbidities, (2) neurologic co-morbidities in medical history, (3) co-morbidity of the motor system which restricts gait, (4) clinical diagnosis of dementia, (5) unable to comply with the test protocol, and (6) severe freezing precluding safe participation.

\section{Baseline characteristics}

General demographic characteristics were assessed by questionnaires. Education level was assessed based on 
the Dutch education system [30] using seven categories $(1=$ less than primary school, $7=$ university degree). The Longitudinal Aging Study Amsterdam Physical Activity Questionnaire (LAPAQ) [31] was completed to obtain physical activity levels of the participants in 2 weeks before assessment, the Mini-Mental State Examination (MMSE) to assess global cognitive functioning [32], and the Fall Efficacy Scale International (FES-I) [33] to assess fear of falling. Fall rate, disease duration, and medication use were assessed by medical history taking of the subjects and proxies, with a fall being defined as "an unexpected event in which the participant comes to rest on the ground, floor or lower level," which is consistent with the recommendations of the Prevention of Falls Network Europe (ProFaNE).

\section{Protocol/procedure}

Participants were instructed to walk back and forth over a course of approximately $8 \mathrm{~m}$ which was marked by a cone at each end and to make wide turns around the cones. During all walking tasks, participants walked at their preferred pace in a quiet room with comfortable footwear. Participants were allowed to use a customary cane, but walkers were not allowed during assessments. Medication was used as normal, and thus, all tests were performed in the on-medication state.

For dual task walking, three different types of tasks were used: walking while (1) counting forward, (2) serially subtracting, and (3) reciting digit spans. These tasks were chosen because it can reasonably be assumed that PFC activity is present during these tasks in PD [2-4, 34-39]. In previous studies, dual task effects were seen on behavioral outcome measures in PD during both walking while serially subtracting [2-4, 34-39] and while reciting digit spans [40]. In addition to these behavioral findings, fNIRS was successfully used in other populations to show increased PFC activity during walking while counting forward [24] and while serially subtracting [24-26].

During walking while counting forward, participants were asked to count forward at their own pace, starting from one. For serial subtraction, participants were instructed to continuously count backward alternating in steps of three or seven, starting from a number between 91 and 100 . When a participant started with serial seven subtractions, we switched to serial three subtractions at the start of the next trial, or when zero was reached. The digit span consisted of repeating series of digits which the assessor said out loud. The number of digits to be repeated was based on the forward digit span of the Wechsler Adult Intelligence Scale (WAIS-III) [41]. Before starting any task, participants sat in a chair and repeated digit spans of increasing length. If subjects were unable to correctly repeat two out of three spans of the same length, the number of digits to be repeated during walking while reciting digit spans was one less than this length. If participants were unable to correctly repeat a span of this length during walking and stopped performing the digit span completely, the span length was reduced until participants did engage in the digit span during walking. This protocol was used to adjust the difficulty of reciting digit spans to each participant's ability, thereby adjusting for baseline differences [40].

Each of the walking tasks was performed five times distributed over five blocks, with each block consisting of three different trials (one of every task). The order of these three trials within a block was randomized. Every trial started with $20 \mathrm{~s}$ of standing still, followed by $40 \mathrm{~s}$ of task performance, and another $20 \mathrm{~s}$ of standing still. During the two 20-s rest periods, participants were instructed to stand as quietly as possible, keep their heads still, look straight ahead, and think of nothing in particular. After $20 \mathrm{~s}$ of standing still, the assessor would say "start" for walking while counting, "start with [first number] minus [three or seven]" for walking while serially subtracting, and "start with [first digit span]" for walking while reciting digit spans. After this start sign, participants were instructed to start walking and simultaneously perform the cognitive task. After $40 \mathrm{~s}$ of task performance, the assessor said "stop," after which the participant had to remain standing quietly where he or she ended for $20 \mathrm{~s}$.

In between trials, participants walked back toward the start position and a rest period of random duration (1-2 min) was given in which instructions for the next trial were given. In between blocks, participants were allowed to sit down and rest until they were ready to continue. Before starting any trial, it was made sure the participant was standing for at least $1 \mathrm{~min}$ to minimize blood pressure fluctuations after standing up.

\section{Feasibility assessment}

To judge the feasibility of the protocol, participants were asked to complete the Borg Rating of Perceived Exertion Scale (RPE) [42], ranging from 6 (very light effort) to 20 (very very hard) after each block. After completion of the protocol, participants were asked to complete a questionnaire about their experience. This questionnaire included: "Did the fNIRS system (Portalites) burden you while walking?" ( 5 point Likert scale: $1=$ "No, not at all" and $5=$ "Yes, a lot") and "Was it doable to complete the protocol?" ( 5 point Likert scale: $1=$ "Yes, very easily doable" and $5=$ "No, undoable").

\section{fNIRS system}

In order to test whether we were able to measure the typical fNIRS signal of neuronal activity, we measured concentration changes in $\mathrm{O}_{2} \mathrm{Hb}$ and $\mathrm{HHb}$ in the PFC with the PortaLite $^{\mathrm{Ts}}$ fNIRS system (Artinis Medical 
Systems, Elst, the Netherlands). Like other fNIRS systems, this system uses near-infrared light which penetrates the skull and brain, but absorbed by hemoglobin $(\mathrm{Hb})$ chromophores in the cortical layer microcirculation. Light was transmitted with two wavelengths, 760 and $850 \mathrm{~nm}$, and data was sampled with a frequency of $10 \mathrm{~Hz}$. The PortaLite $^{\text {ma }}$ uses wireless technology (Bluetooth), allowing participants to walk without restriction of wires. Two devices were placed on the forehead of the participants, one on the right and the other on the left side. Both devices were positioned at a height of $15 \%$ of the nasion-inion distance from nasion, and we placed the middle of the device at $7 \%$ of the head circumference to the left and right from midline, to avoid measuring the midline sinus. These locations roughly target left and right Brodmann's areas 9 and 10, which represent the dorsolateral and anterior PFC [43, 44]. The devices were shielded from ambient light by covering the whole forehead with a black cloth. Oxysoft version 3.0.52 (Artinis Medical Systems, Elst, The Netherlands) was used for data collection.

Based on different $\mathrm{Hb}$ absorption spectra, concentration changes of $\mathrm{O}_{2} \mathrm{Hb}$ and $\mathrm{HHb}$ in the targeted PFC area were calculated from the changes in detected light intensity. This was done using the modified Lambert-Beer law, assuming constant light scattering [45]. A PortaLite ${ }^{\text {Tu }}$ has three transmitters and one receiver, with transmitterreceiver distances of 30,35 , and $40 \mathrm{~mm}$. The differential path length factor (DPF), which accounts for the increased distance traveled by light due to scattering, was set to 6 for all participants. This is in line with previous studies in adults [46]. Although the DPF is age-dependent [46], no data is available on the actual DPF variation in adults older than 50. With the fixed DPF we chose, the assessment of relative changes in $\mathrm{O}_{2} \mathrm{Hb}$ and $\mathrm{HHb}$ within and between tasks will not be affected.

\section{fNIRS analysis}

Measured concentrations of $\mathrm{O}_{2} \mathrm{Hb}$ and $\mathrm{HHb}$ from each of the Portalite devices were exported to MATLAB (MATLAB and Statistics Toolbox Release 2012b, The MathWorks, Inc., Natick, Massachusetts, United States), in which further data processing was done. First, $\mathrm{O}_{2} \mathrm{Hb}$ and $\mathrm{HHb}$ signals of the three channels (the three transmitter-receiver distances) per PortaLite were averaged. Then, the moving standard deviation-based artifact removal (movement artifact reduction algorithm-MARA) method was performed within each trial [47]. The threshold for artifact detection was set to 0.45 for $\mathrm{O}_{2} \mathrm{Hb}$ and 0.18 for $\mathrm{HHb}$, with a window length for moving standard deviation calculation at $0.5 \mathrm{~s}$, and a window length for artifact correction (LOESS smoothing window) on $1 \mathrm{~s}$. These parameters were chosen after testing several settings and visual inspection of the data by JC and FN on adequate removal of large (movement) artifacts while not affecting physiological fluctuations. Next, the fNIRS signals were linearly detrended per trial and low-pass filtered at $0.1 \mathrm{~Hz}$ using a Butterworth filter to remove heart rate and other higher frequency physiological signals. To enable direct comparison of the five different trials within each task, the filtered signals were biased, using the average concentration of the $5 \mathrm{~s}$ before the "Start" instruction as reference (zero). Then, individual trials were averaged per task to create three mean time course signals per person, which were then averaged over all participants (see Fig. 1). Finally, the mean concentrations $\left(\mathrm{O}_{2} \mathrm{Hb}\right.$ and $\left.\mathrm{HHb}\right)$ during the final $5 \mathrm{~s}$ of all 20 -s rest periods were calculated over all trials for all participants and mean concentrations $\left(\mathrm{O}_{2} \mathrm{Hb}\right.$ and $\left.\mathrm{HHb}\right)$ of the $40 \mathrm{~s}$ after the "Start" instruction were calculated for each trial and then averaged for each of the three walking tasks.

\section{Gait performance analysis}

To be able to judge whether our protocol was able to detect behavioral dual task effects and to interpret the level of PFC activity in relation to behavioral performance, gait parameters, and performance on the cognitive tasks were measured.

Walking performance parameters were measured on a GAITRite ${ }^{\oplus}$ mat (CIR Systems Inc., Clifton, NJ 07012, USA), over which the participants walked while walking forth over the $8-\mathrm{m}$ course. The GAITRite ${ }^{\bullet}$ mat is an electronic roll-up walkway with pressure sensors embedded in a carpet. The carpet is $5.18-\mathrm{m}$ long and $0.90-\mathrm{m}$ wide, and the active area is $4.27-\mathrm{m}$ long and $0.61-\mathrm{m}$ wide. The mat is connected to a personal computer using GAITRite software version 4.0 (CIR Systems Inc., Clifton, NJ 07012, USA) for processing and storing the data. This system has been shown to be reliable and accurate for measuring walking parameters in elderly and in people with $\mathrm{PD}$ [48-50]. For investigating gait under dual task conditions in PD, it has been used as reference [51]. Automatic identification of footsteps was checked step by step for each trial and manually corrected where necessary. Walking performance parameters included gait speed, cadence, stride length, stride time, and the coefficient of variability $(\mathrm{CV})$ of both stride length and stride time calculated as (standard deviation/mean)* 100 . Gait performance outcome measures were determined for individual trials first (with an average of $13.1 \pm 2.9$ footsteps) and then averaged over all trials within the three tasks for further analysis.

\section{Cognitive performance analysis}

Cognitive performance measures included the number of subtractions and digit spans completed within the $40 \mathrm{~s}$ of task performance and the percentage of correct 


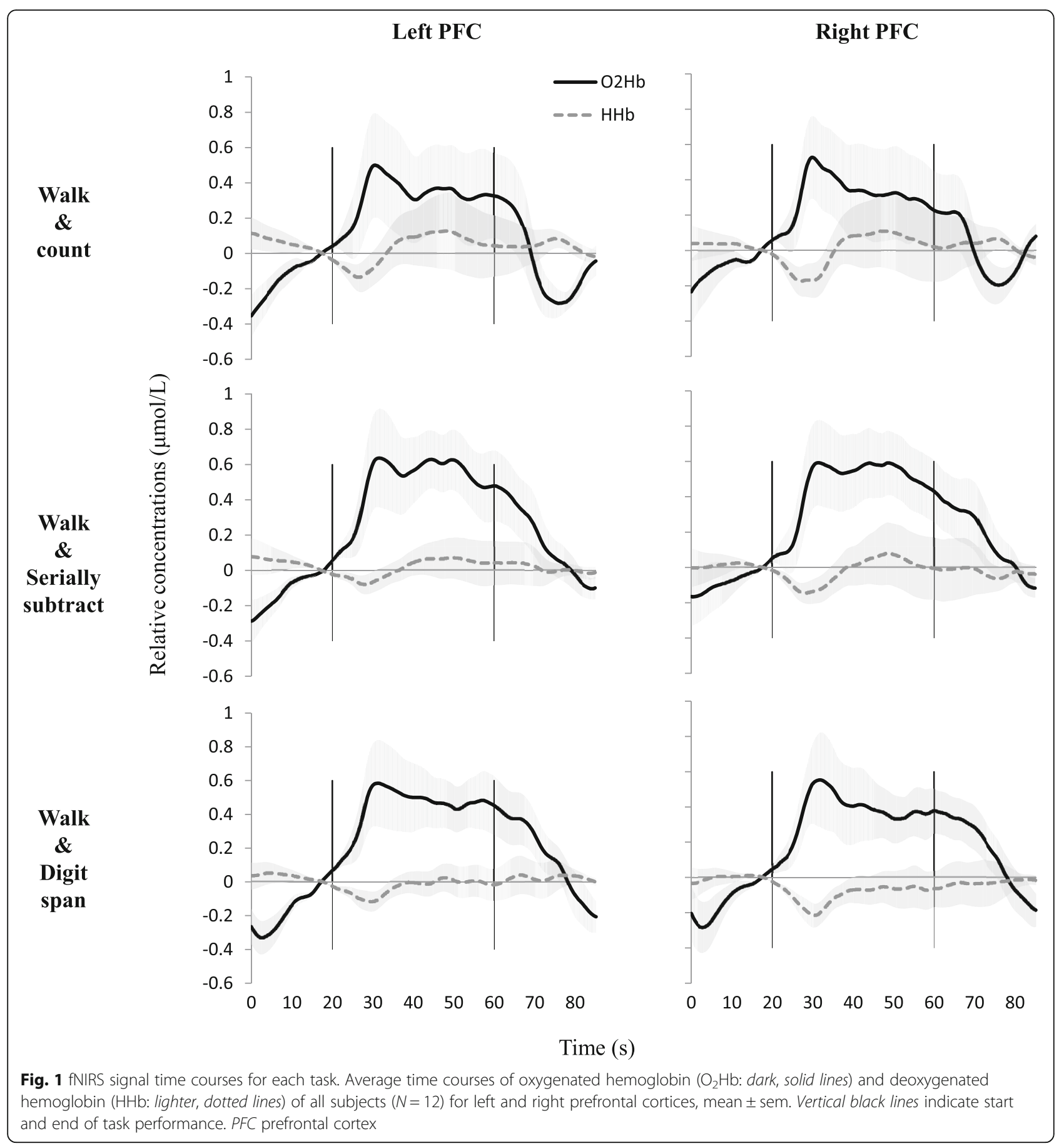

answers on both tasks. Outcome measures on gait and the cognitive tasks were determined for individual trials first and then averaged over all trials within the three tasks for further analysis.

\section{Statistical analysis}

Statistical analysis was performed using IBM SPSS Statistics Version 21. To estimate the level of activity and noise, we calculated group means and $95 \%$ confidence intervals $(\mathrm{CI})$ for both $\mathrm{O}_{2} \mathrm{Hb}$ and $\mathrm{HHb}$ concentrations. Likewise, means and $95 \%$ confidence intervals were calculated for the behavioral performance measures.

To explore differences between tasks in $\mathrm{O}_{2} \mathrm{Hb}$ and $\mathrm{HHb}$ concentrations and behavioral performance measures, we calculated Cohen's $d_{\mathrm{z}}$ effect sizes based on mean difference scores [52]. Effect sizes of 0.2, 0.5, and 0.8 were interpreted as small, medium, and large, respectively [53]. Differences between dual walking tasks were tested for 
significance with the Wilcoxon signed-rank test, with $P<.05$ as threshold for statistical significance.

\section{Results \\ Participants}

After screening, 14 patients were found eligible and were included in the study. One of these patients was unable to safely perform the tests, and another patient came in tired and stopped after two blocks because of fatigue. These two participants (both male; 78 and 76 years old; 8 and 11 years since PD diagnosis; Hoehn and Yahr stage 2 and 2.5; 6 and 0 falls in the previous 6 months) were excluded from further analysis. Characteristics of the remaining 12 participants are shown in Table 1 . For these participants, a total of 155 out of 180 intended trials were included in analyses. Reasons for dropped trials were inconsistent use of walking aid or inconsistent length of digit spans to be recited. No trials were dropped because of noisy fNIRS signals due to movement artifacts. For all participants, at least three trials per task were included in the analyses. Only one participant used a cane during the trials that were included in analyses.

\section{Feasibility analysis}

Two of the initial 14 participants were unable to complete the whole protocol (see above). The 12 participants included in the study reported that it was doable to complete the full protocol (median Likert-scale score 1 , range 1 (very easily doable) to 3 (neutral)). On average, the participants scored $10.6 \pm 1.6$ on the Borg-RPE, corresponding to fairly light effort. Concerning the two Portalite devices placed on their forehead, participants reported that these did not burden them during walking

Table 1 Characteristics of participants $(N=12)$

\begin{tabular}{|c|c|}
\hline Age (years) & $70.1 \pm 5.4$ \\
\hline Gender (men) & 7 \\
\hline Years since diagnosis PD & $5.7 \pm 3.3$ \\
\hline Hoehn and Yahr stage (2/2.5/3) & $4 / 5 / 3$ \\
\hline Walking aid in daily life (yes/no) & $3 / 9$ \\
\hline Number of falls in previous 6 months $(0 / 1 / 2 / 7)$ & $5 / 2 / 4 / 1$ \\
\hline Education (possible range $1-7)^{\mathrm{a}}$ & $5.7 \pm 1.3$ \\
\hline MMSE (possible range $0-30)^{b}$ & $27.4 \pm 2.0$ \\
\hline LAPAQ (kcals/day) & $630.3 \pm 712.1$ \\
\hline FES-I (possible range $16-64)^{c}$ & $29.3 \pm 8.2$ \\
\hline Number of different medications used & $5.3 \pm 2.7$ \\
\hline
\end{tabular}

Values are mean \pm standard deviation or frequency

PD Parkinson's disease, MMSE Mini-Mental State Examination, LAPAQ Longitudinal Aging Study Amsterdam Physical Activity Questionnaire, FES-I Fall Efficacy

Scale International

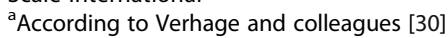

${ }^{b}$ Higher scores indicating better global cognitive functioning

${ }^{\mathrm{c}}$ Higher scores indicating more fear of falling (median Likert-scale score 1, range 1 (no burden at all) to 2 (no burden)).

\section{Prefrontal cortical activity PFC activity (group level)}

Figure 1 shows the group averaged time course of $\mathrm{O}_{2} \mathrm{Hb}$ and $\mathrm{HHb}$ concentrations in bilateral PFC during the three dual walking tasks. In all tasks, the average $\mathrm{O}_{2} \mathrm{Hb}$ concentrations increased from resting conditions after starting the task and decreased again during rest after task performance. $\mathrm{HHb}$ concentrations remained relatively stable or showed slight reductions during task performance when compared to rest. Thus, in all three dual walking tasks, bilateral PFC activity patterns were found on a group level.

Mean $\mathrm{O}_{2} \mathrm{Hb}$ and $\mathrm{HHb}$ concentrations in left and right PFC during the three dual walking tasks are shown in Table 2 (also see Additional file 1: Figure S1). During walking while serially subtracting and walking while reciting digit spans, mean $\mathrm{O}_{2} \mathrm{Hb}$ concentrations in left and right PFC were significantly higher than those during rest (lower limit $95 \% \mathrm{CI}>0$ ). Mean $\mathrm{HHb}$ concentrations were similar between walking tasks and rest $(95 \% \mathrm{CI}$ includes 0 ). No significant differences between tasks were seen in mean $\mathrm{O}_{2} \mathrm{Hb}$ or $\mathrm{HHb}$ concentrations in left or right PFC. Effect sizes of the concentration differences between dual walking tasks were small (Table 3).

\section{PFC activity (individual level)}

The $\mathrm{O}_{2} \mathrm{Hb}$ and $\mathrm{HHb}$ signals were stable over the five trials within participants (see Additional file 1: Figures S3-S7), which indicates that the wireless fNIRS system reliably measures PFC activity within subjects. However, we found large variability between participants (see standard errors in Fig. 1 and confidence intervals in Table 2). Therefore, we explored differences in individual fNIRS signal patterns and were able to identify five subgroups of participants based on signal patterns. The authors FN and JC both independently inspected the figures of each subject (individual trials and average), which resulted in two identical group distributions. Five participants had signals as hypothesized (classic task related hemodynamic response signal), an increase in $\mathrm{O}_{2} \mathrm{Hb}$, and slight decrease or stable $\mathrm{HHb}$ during task performance (see Additional file 1: Figure S3). One participant showed an increased $\mathrm{O}_{2} \mathrm{Hb}$ with stable $\mathrm{HHb}$, but only at the start of task performance (Additional file 1: Figure S4). Three participants were identified as non-responders, where both $\mathrm{O}_{2} \mathrm{Hb}$ and $\mathrm{HHb}$ remained relatively stable during task performance and for all task repetitions (Additional file 1: Figure S5). Two participants showed an inverse response during walking while counting; $\mathrm{O}_{2} \mathrm{Hb}$ decreased, while $\mathrm{HHb}$ remained relatively stable or decreased slightly (Additional file 1: Figure S6). During walking while serially 
Table 2 Concentrations of $\mathrm{O}_{2} \mathrm{Hb}$ and $\mathrm{HHb}$ and gait performance measures during all three walking tasks

\begin{tabular}{llll}
\hline & Walking while counting & Walking while serially subtracting & Walking while reciting digit spans \\
\hline fNIRS & & & $0.44(0.09-0.78)$ \\
$\mathrm{O}_{2} \mathrm{Hb}$ left $(\mu \mathrm{mol} / \mathrm{L})$ & $0.32(-0.18-0.82)$ & $0.49(0.14-0.84)$ & $0.36(0.03-0.70)$ \\
$\mathrm{O}_{2} \mathrm{Hb}$ right $(\mu \mathrm{mol} / \mathrm{L})$ & $0.31(-0.12-0.75)$ & $0.46(0.12-0.81)$ & $-0.03(-0.16-0.10)$ \\
$\mathrm{HHb}$ left $(\mu \mathrm{mol} / \mathrm{L})$ & $0.03(-0.28-0.33)$ & $0.01(-0.15-0.17)$ & $-0.10(-0.24-0.05)$ \\
$\mathrm{HHb}$ right $(\mu \mathrm{mol} / \mathrm{L})$ & $0.00(-0.28-0.28)$ & $-0.02(-0.25-0.21)$ & $79.9(68.2-91.6)$ \\
Gait performance & & & $94.0(84.0-104.0)$ \\
Gait speed $(\mathrm{cm} / \mathrm{s})$ & $83.7(73.7-93.7)$ & $82.3(71.8-92.9)$ & $101.2(91.5-111.0)^{* *}$ \\
Cadence $(\mathrm{steps} / \mathrm{min})$ & $94.6(86.2-103)$ & $93.2(84.6-101.9)$ & $5.4(4.4-6.5)^{*}$ \\
Stride length $(\mathrm{cm})$ & $106.3(97.7-114.9)$ & $106(96.6-115.4)$ & $1.3(1.2-1.5)$ \\
Stride length variability $(\%)$ & $4.2(3.2-5.2)$ & $4.5(2.9-6.2)$ & $3.6(2.7-4.6)$ \\
Stride time $(\mathrm{s})$ & $1.3(1.2-1.4)$ & $1.3(1.2-1.4)$ & $4.0(2.9-5.2)$ \\
Stride time variability $(\%)$ & $4.2(2.4-5.9)$ & & \\
\hline
\end{tabular}

Values are mean (95\% confidence intervals)

* $P<.05$ compared to walking while counting

${ }^{*} P<.05$ compared to both walking while talking and serially subtracting

subtracting and walking while reciting digit spans, these two participants showed no inverse response but slight increases in or stable $\mathrm{O}_{2} \mathrm{Hb}$ concentrations. Finally, one participant showed a highly unexpected response, with an initial increase in $\mathrm{O}_{2} \mathrm{Hb}$ and increase in $\mathrm{HHb}$ in the second half of task performance (Additional file 1: Figure S7). These subgroups could not be identified based on participant characteristics or task performance data, in which no obvious differences between subgroups were present.

\section{Behavioral performance}

Gait performance during the dual walking tasks is shown in Table 2 (also see Additional file 1: Figure S2). For gait speed, cadence, stride time, and stride time variability, no significant differences in gait performance between any of the dual walking tasks were found. During walking while reciting digit spans, participants showed significantly decreased stride length when compared to walking while counting $(z=-2.7, P=.006)$ and serially subtracting $(z=-2.1, P=.034)$ and increased stride length variability when compared to walking while counting $(z=-2.6, P=.010)$. In addition to these significant differences, for which effect sizes were medium to large (Table 3), a medium effect size (Cohen's $d_{z}=0.72$ ) was found for gait speed during walking while counting versus reciting digit spans. All other effect sizes were small (Table 3).

During walking while serially subtracting, participants gave an average of 25.3 (95\% CI 19.7-30.8) answers, of which on average, $98.4 \%$ (95\% CI 97.5-99.3) was

Table 3 Effect sizes (Cohen's $d_{z}$ ) between the walking tasks for both concentrations of $\mathrm{O} 2 \mathrm{Hb}$ and $\mathrm{HHb}$ and gait performance

\begin{tabular}{llll}
\hline & $\begin{array}{l}\text { Walking while serially } \\
\text { subtracting }>\text { counting }\end{array}$ & $\begin{array}{l}\text { Walking while reciting } \\
\text { digit spans }>\text { counting }\end{array}$ & $\begin{array}{c}\text { Walking while serially subtracting }> \\
\text { reciting digit spans }\end{array}$ \\
\hline fNIRS & .29 & .08 & .36 \\
$\mathrm{O}_{2} \mathrm{Hb}$ left & .31 & .20 & .27 \\
$\mathrm{O}_{2} \mathrm{Hb}$ right & -.13 & -.36 & .43 \\
$\mathrm{HHb}$ left & -.07 & -.18 & .42 \\
$\mathrm{HHb}$ right & & & .40 \\
Gait performance & -.22 & -.72 & -.23 \\
Gait speed (m/s) & -.33 & -.15 & .73 \\
Cadence (steps/min) & -.09 & -.93 & -.32 \\
Stride length (m) & .11 & $\mathbf{1 . 0 4}$ & .18 \\
Stride length variability (\%) & .30 & .16 & .24 \\
Stride time (s) & -.05 & -.20 & \\
Stride time variability (\%) & & & \\
\hline
\end{tabular}


correct. The mean length of digit spans to be repeated during walking while reciting digit spans was 4.9 digits (95 \% CI 4.0-5.8). Two participants were unable to perform the predetermined length of digit spans during walking; for them, the number of digits was reduced until they were able to perform (from 6 to 5 and from 7 to 4 digits). During walking while reciting digit spans, participants recited an average of 6.0 (95\% CI 5.2-6.8) spans, with a mean success rate of $88.4 \%(95 \% \mathrm{CI}$ 79.6-97.3).

\section{Discussion}

In this pilot study, we aimed to examine the feasibility of measuring PFC activity during dual task walking in patients with PD with use of a portable fNIRS device. Good feasibility of the portable fNIRS device was demonstrated by the fact that most participants experienced a low burden of the two fNIRS devices placed on the forehead during walking, were able to perform the different dual task walking paradigms, and reported that it took them little effort to complete the full protocol. Importantly, when averaged over all participants, fNIRS showed typical cortical activity patterns of increased oxygenated $\left(\mathrm{O}_{2} \mathrm{Hb}\right)$ and stable deoxygenated hemoglobin $(\mathrm{HHb})$ concentrations during the three dual walking tasks when compared to rest. This further supports feasibility of using portable fNIRS to measure PFC activity during dual task walking in PD.

These findings are in line with previous studies in which fNIRS was successfully used to measure PFC activity during dual task walking in healthy young adults [24-26] and elderly [22, 23, 54]. In contrast to these studies that found differences in PFC activity levels between walking tasks, effect sizes in our study were small. Although we were able to measure PFC activity during dual task walking, future protocols need to be improved to enable detection of differences between tasks. This can possibly be done by either reducing variability in concentration measures (i.e., reducing noise), especially between participants, or choosing different tasks. Below, we will elaborate on these issues and propose protocol improvements for future work.

Large variability occurred mainly between participants, while time courses of $\mathrm{O}_{2} \mathrm{Hb}$ and $\mathrm{HHb}$ were highly stable within participants. The first potential cause of such inter-individual differences is the fNIRS optode placement on the forehead. Although the placement procedure was identical for all participants, it was based on relative distances from external landmarks (nasion and inion). It is possible that this method resulted in the targeting of slightly different brain areas due to morphological differences between subjects $[19,55]$. To avoid targeting different brain areas, it could be beneficial to relocate the devices until task-related cortical activity is seen before starting measurements. The window in which to move the devices should however be limited, so that after relocation, still the region of interest is likely targeted. Also, the typically expected response of the targeted brain area should be very well known and distinct from directly surrounding brain areas [19]. Another solution could be to use portable multichannel fNIRS systems. When available, such a system could cover the whole PFC, ensuring that the target area is within the field of view $[56,57]$. Another option is to use structural magnetic resonance imaging or transcranial magnetic stimulation before placing fNIRS probes to enable more precise placement over a target area, although these are costly and laborious solutions [19]. A second cause of individual differences can be systemic task responses (see also our discussion on the study limitations) such as changes in blood pressure or heart rate [58]. Adding continuous blood pressure and heart rate monitoring can help to determine the role of systemic responses in individual differences.

Future studies should try to limit individual differences by optimizing protocols as suggested above. When still present, causes of individual differences should be further investigated and taken into account when determining sample sizes and when interpreting group averaged data. Only in the case of obvious measurement errors should a participant who shows non-hypothesized fNIRS signals be excluded from analyses. For example, when it can reasonably be assumed that the wrong cortical area was targeted, when the data contains too much noise due to movement artifacts, or when systemic changes in blood pressure and subsequent changes in cerebral perfusion affect the hemodynamic response to activity, there are objective arguments to exclude a subject.

Apart from inter-individual differences, the choice of walking tasks is an important topic for improvement of future studies. First, although simple counting during walking offers a controlled condition and prevents that participants' thoughts start to wander ("daydreaming"), it might be a rather difficult dual task itself. Accordingly, in healthy young participants walking while counting led to increased PFC activity when compared to usual walking [24]. The dual tasks we used might thus not have differed much in terms of difficulty, which would explain the lack of differences in PFC activity levels between tasks. This is further supported by the similar gait performance we saw between walking while counting and serially subtracting. And, although we did not measure it as a single task, good performances on both serially subtracting and reciting digit spans while walking suggest that these tasks might not have been much more difficult than walking while counting. Future studies should use tasks that differ more in terms of difficulty, such as usual walking without any secondary task. With usual walking as a reference task, previous studies found relatively 
increased PFC activity during dual tasks like walking while talking [22, 23], serially subtracting [24-26], and balancing a ping pong ball on a card [29]. A second consideration regarding the choice of task is the use of walking while reciting digit spans. Effect sizes of the difference with walking while counting for gait speed, stride length, and stride length variability were moderate to large. However, defining the number of digits to be recited was not straightforward as reflected by the two participants who were unable to perform the predefined length while walking. Also, citing the numbers seemed to provide participants with an external rhythm on which they paced their walking. Finally, walking while serially subtracting showed larger differences (effect sizes) with walking while counting for $\mathrm{O}_{2} \mathrm{Hb}$ concentration change. Using walking while reciting digit spans in future fNIRS dual task work is thus not recommended.

A limitation of the present study was the small sample size. Although the sample size was sufficient to fulfill our primary aims regarding feasibility, it might have been too low to find differences between dual walking tasks. Based on the variability and mean difference in $\mathrm{O}_{2} \mathrm{Hb}$ concentration between walking while counting and walking while serially subtracting in the present study, 85 participants would be needed to find significant differences in $\mathrm{O}_{2} \mathrm{Hb}$ concentrations between these tasks (power $=0.80$, alpha $=$ 0.05 , two-tailed testing). However, improving protocols as suggested, to reduce variability and increase contrasts between tasks, will reduce this required sample size. Indeed, our recently published work shows that groups of $68 \mathrm{PD}$ patients and 38 healthy controls were sufficient to find $\mathrm{O}_{2} \mathrm{Hb}$ concentration differences between complex walking tasks within groups [13].

Also, we cannot generalize findings to the broader PD population. Although all participants were identified with an increased risk of falling, they were able to walk at least 5 min unassisted, their cognitive functioning was relatively well, and all were in mild to moderate stages of PD. Two patients who had passed screening for inclusion and exclusion criteria were unable to complete the protocol. Although no large differences were present, these two participants were slightly older and had a longer history of PD than the mean of those participants who were able to complete the protocol. This raises doubt whether the protocol is feasible for more frail and severely affected PD patients, which is a subject for future research.

Other limitations concern our use of fNIRS. We did not control for superficial (e.g., skin) hemodynamics $[59,60]$, for example, by using a short reference channel [59]. Thus, we cannot rule out the influence of blood flow through skin or the occipitofrontalis muscle, which might have increased during task performance [61]. However, in frontal brain areas, high correlations were found between fNIRS signals and fMRI signals from the cortical gray matter layer while performing several cognitive tasks $[62,63]$. These correlations were higher than correlations between fNIRS signals and soft tissue fMRI signals [62]. Although our fNIRS signals are likely affected by skin blood flow, we can still assume based on these data that they do reflect cortical neuronal activity. A further limitation is that only PFC activity levels were measured. We did not measure any other cortical areas to ensure specificity of signals to PFC instead of whole brain effects. The use of a multiple channel fNIRS system would allow for measurements of multiple cortical areas and thereby to differentiate between region-specific and global effects; however, the increase in weight of such a system may reduce the feasibility. Besides cognitive tasks, postural changes and walking exercise may all lead to changes in blood pressure which in turn can affect global cerebral blood flow [18]. For this reason, it is advisable to measure blood pressure simultaneously with fNIRS.

\section{Conclusions}

In this study, we showed that using two small, lightweight, portable fNIRS devices placed on the forehead is feasible to measure PFC activity during dual task walking in PD patients. We provided recommendations for improvements of protocols to increase the sensitivity to detect differences in PFC activity and behavioral performance between dual walking tasks and potentially between populations (PD patients vs. healthy age-matched controls) and/or changes over time (neurorehabilitation training effects). With improved protocols, portable fNIRS seems to be a very promising tool to further study the role of the PFC in mechanisms underlying difficulties in dual task walking in PD.

\section{Additional file}

Additional file 1: Supplementary figures can be found in the document "Additional file 1-Supplementary figures.pdf" (PDF $1324 \mathrm{~kb}$ )

\section{Abbreviations \\ CV: Coefficient of variability; DPF: Differential path length factor; \\ EEG: Electroencephalography; FES-I: Fall Efficacy Scale International; \\ fMRI: Functional magnetic resonance imaging; fNIRS: Functional near-infrared spectroscopy; Hb: Hemoglobin; HHb: Deoxygenated hemoglobin; LAPAQ: Longitudinal Aging Study Amsterdam Physical Activity Questionnaire; MARA: Movement artifact reduction algorithm; MMSE: Mini- Mental State Examination; $\mathrm{O}_{2} \mathrm{Hb}$ : Oxygenated hemoglobin; PD: Parkinson's disease; PFC: Prefrontal cortex; RPE: Rating of perceived exertion; \\ WAIS: Wechsler Adult Intelligence Scale}

\section{Acknowledgements}

We thank the participants in this study and Malou H.J. Fanchamps for her work on the study during her internship at the department of Geriatric Medicine of the Radboud University Medical Center, the Netherlands.

\section{Funding}

The study was partially funded by the European Union 7th framework project V-TIME (278169 FP7). 


\section{Availability of data and materials}

The dataset supporting the conclusions of this article is available via the corresponding author (JC, jurgen.claassen@radboudumc.nl).

\section{Authors' contributions}

All authors contributed to the concept and design of the present study. Acquisition of data was done by MR and FN, analysis by MR, JC, MM and FN, and interpretation by all authors. The manuscript was drafted mainly by FN, with substantial contribution of MR and JC. All authors critically revised the draft version and gave approval for submission.

\section{Competing interests}

The authors declare that they have no competing interests.

\section{Ethics approval and consent to participate}

All participants participated voluntarily and gave their written informed consent prior to the start of the study. The study was approved by the ethical committee "CMO regio Arnhem-Nijmegen" and was performed according to the principles of the Declaration of Helsinki.

\section{Author details}

'Donders Institute for Brain, Cognition and Behaviour, Nijmegen, The Netherlands. ${ }^{2}$ Departments of Neurology, Geriatric Medicine, and Radboud Alzheimer Center, Radboud University Medical Center, Nijmegen, The Netherlands. ${ }^{3}$ Department of Neurology, Center for the study of Movement Cognition, and Mobility, Tel Aviv Sourasky Medical Center, Tel Aviv, Israel. ${ }^{4}$ Sackler Faculty of Medicine, Tel Aviv University, Tel Aviv, Israel. ${ }^{5}$ EuroMov, University of Montpellier, Montpellier, France.

Received: 30 June 2016 Accepted: 16 September 2016 Published online: 23 September 2016

\section{References}

1. Kelly VE, Eusterbrock AJ, Shumway-Cook A. A review of dual-task walking deficits in people with Parkinson's disease: motor and cognitive contributions, mechanisms, and clinical implications. Parkinsons Disease. 2012;2012:918719.

2. Stegemoller EL, Wilson JP, Hazamy A, Shelley MC, Okun MS, Altmann LJ, Hass CJ. Associations between cognitive and gait performance during single- and dual-task walking in people with Parkinson disease. Phys Ther. 2014;94:757-66.

3. Wild LB, De Lima DB, Balardin JB, Rizzi L, Giacobbo BL, Oliveira HB, De Lima II A, Peyre-Tartaruga LA, Rieder CR, Bromberg E. Characterization of cognitive and motor performance during dual-tasking in healthy older adults and patients with Parkinson's disease. J Neurol. 2013:260:580-9.

4. Yogev G, Giladi N, Peretz C, Springer S, Simon ES, Hausdorff JM. Dual tasking, gait rhythmicity, and Parkinson's disease: which aspects of gait are attention demanding? Eur J Neurosci. 2005;22:1248-56.

5. Plotnik M, Giladi N, Dagan Y, Hausdorff JM. Postural instability and fall risk in Parkinson's disease: Impaired dual tasking, pacing and bilateral coordination of gait during the "ON" medication state. Mov Disord. 2011;26:S159.

6. Yogev-Seligmann G, Hausdorff JM, Giladi N. The role of executive function and attention in gait. Mov Disord. 2008;23:329-42.

7. Nutt JG, Horak FB, Bloem BR. Milestones in gait, balance, and falling. Mov Disord. 2011;26:1166-74

8. Hausdorff JM, Doniger GM, Springer S, Yogev G, Simon ES, Giladi N. A common cognitive profile in elderly fallers and in patients with Parkinson's disease: the prominence of impaired executive function and attention. Exp Aging Res. 2006:32:411-29.

9. Kudlicka A, Clare L, Hindle JV. Executive functions in Parkinson's disease: systematic review and meta-analysis. Mov Disord. 2011;26:2305-15.

10. Williams-Gray CH, Evans JR, Goris A, Foltynie T, Ban M, Robbins TW, Brayne C, Kolachana BS, Weinberger DR, Sawcer SJ, Barker RA. The distinct cognitive syndromes of Parkinson's disease: 5 year follow-up of the CamPalGN cohort. Brain. 2009;132:2958-69.

11. Bohnen NI, Jahn K. Imaging: what can it tell us about parkinsonian gait? Mov Disord. 2013;28:1492-500.

12. Amboni M, Barone $P$, Hausdorff JM. Cognitive contributions to gait and falls: evidence and implications. Mov Disord. 2013;28:1520-33.

13. Maidan I, Nieuwhof F, Bernad-Elazari H, Reelick MF, Bloem BR, Giladi N, Deutsch JE, Hausdorff JM, Claassen JA, Mirelman A. The role of the frontal lobe in complex walking among patients with Parkinson's disease and healthy older adults: an fNIRS study. Neurorehabil Neural Repair. 2016.

14. Cutini S, Brigadoi S. Unleashing the future potential of functional near-infrared spectroscopy in brain sciences. J Neurosci Methods. 2014;232:152-6.

15. Obrig H. NIRS in clinical neurology-a 'promising' tool? Neuroimage. 2014;85(Pt 1):535-46.

16. Ferrari M, Quaresima V. A brief review on the history of human functional near-infrared spectroscopy (fNIRS) development and fields of application. Neuroimage. 2012;63:921-35.

17. Perrey S. Possibilities for examining the neural control of gait in humans with fNIRS. Front Physiol. 2014;5:204.

18. Scholkmann F, Kleiser S, Metz AJ, Zimmermann R, Mata Pavia J, Wolf U, Wolf M A review on continuous wave functional near-infrared spectroscopy and imaging instrumentation and methodology. Neuroimage. 2014;85(Pt 1):6-27.

19. Leff DR, Orihuela-Espina F, Elwell CE, Athanasiou T, Delpy DT, Darzi AW, Yang GZ. Assessment of the cerebral cortex during motor task behaviours in adults: a systematic review of functional near infrared spectroscopy (fNIRS) studies. Neuroimage. 2011;54:2922-36.

20. Handojoseno AMA, Shine JM, Nguyen TN, Tran Y, Lewis SJG, Nguyen HT. Analysis and prediction of the freezing of gait using EEG brain dynamics. IEEE Trans Neural Syst Rehabil Eng. 2015;23:887-96.

21. Perrey S. Non-invasive NIR spectroscopy of human brain function during exercise. Methods. 2008;45:289-99.

22. Holtzer R, Mahoney JR, Izzetoglu M, Izzetoglu K, Onaral B, Verghese J. fNIRS study of walking and walking while talking in young and old individuals. J Gerontol A Biol Sci Med Sci. 2011;66:879-87.

23. Holtzer R, Mahoney JR, Izzetoglu M, Wang C, England S, Verghese J. Online fronto-cortical control of simple and attention-demanding locomotion in humans. Neuroimage. 2015;112:152-9.

24. Mirelman A, Maidan I, Bernad-Elazari H, Nieuwhof F, Reelick M, Giladi N, Hausdorff JM. Increased frontal brain activation during walking while dual tasking: an fNIRS study in healthy young adults. J Neuroeng Rehabil. 2014;11:85.

25. Meester D, Al-Yahya E, Dawes H, Martin-Fagg P, Pinon C. Associations between prefrontal cortex activation and $\mathrm{H}$-reflex modulation during dual task gait. Front Hum Neurosci. 2014;8.

26. Lu CF, Liu YC, Yang YR, Wu YT, Wang RY. Maintaining gait performance by cortical activation during dual-task interference: a functional near-infrared spectroscopy study. PLOS ONE. 2015;10:e0129390.

27. Mirelman A, Rochester $L$, Reelick M, Nieuwhof F, Pelosin E, Abbruzzese G, Dockx K, Nieuwboer A, Hausdorff JM. V-TIME: a treadmill training program augmented by virtual reality to decrease fall risk in older adults: study design of a randomized controlled trial. BMC Neurol. 2013;13:15.

28. Mirelman A, Rochester L, Maidan I, Del Din S, Alcock L, Nieuwhof F, Rikkert MO, Bloem BR, Pelosin E, Avanzino L, et al. Addition of a non-immersive virtual reality component to treadmill training to reduce fall risk in older adults (V-TIME): a randomised controlled trial. Lancet. 2016.

29. Atsumori H, Kiguchi M, Katura T, Funane T, Obata A, Sato H, Manaka T, Iwamoto M, Maki A, Koizumi H, Kubota K. Noninvasive imaging of prefrontal activation during attention-demanding tasks performed while walking using a wearable optical topography system. J Biomed Opt. 2010:15:046002

30. Verhage F. Intelligentie en leeftijd: onderzoek bij Nederlanders van twaalf tot zevenenzeventig jaar. Assen: Van Gorcum; 1964.

31. Stel VS, Smit JH, Pluijm SM, Visser M, Deeg DJ, Lips P. Comparison of the LASA physical activity questionnaire with a 7-day diary and pedometer. J Clin Epidemiol. 2004;57:252-8.

32. Folstein MF, Folstein SE, McHugh PR. "Mini-mental state". A practical method for grading the cognitive state of patients for the clinician. J Psychiatr Res. 1975;12:189-98.

33. Yardley L, Beyer N, Hauer K, Kempen G, Piot-Ziegler C, Todd C. Development and initial validation of the Falls Efficacy Scale-International (FES-I). Age Ageing. 2005:34:614-9.

34. Hausdorff JM, Balash J, Giladi N. Effects of cognitive challenge on gait variability in patients with Parkinson's disease. J Geriatr Psychiatry Neurol. 2003; 16:53-8.

35. Yogev G, Plotnik M, Peretz C, Giladi N, Hausdorff JM. Gait asymmetry in patients with Parkinson's disease and elderly fallers: when does the bilateral coordination of gait require attention? Exp Brain Res. 2007;177:336-46. 
36. Brown LA, De Bruin N, Doan JB, Suchowersky O, Hu B. Novel challenges to gait in Parkinson's disease: the effect of concurrent music in single- and dual-task contexts. Arch Phys Med Rehabil. 2009;90:1578-83.

37. Plotnik M, Giladi N, Dagan Y, Hausdorff JM. Postural instability and fall risk in Parkinson's disease: impaired dual tasking, pacing, and bilateral coordination of gait during the "ON" medication state. Exp Brain Res. 2011;210:529-38.

38. Plotnik M, Giladi N, Hausdorff JM. Bilateral coordination of gait and Parkinson's disease: the effects of dual tasking. J Neurol Neurosurg Psychiatry. 2009;80:347-50.

39. Panyakaew $P$, Bhidayasiri R. The spectrum of preclinical gait disorders in early Parkinson's disease: subclinical gait abnormalities and compensatory mechanisms revealed with dual tasking. J Neural Transm. 2013;120:1665-72.

40. Rochester L, Galna B, Lord S, Burn D. The nature of dual-task interference during gait in incident Parkinson's disease. Neuroscience. 2014;265:83-94.

41. Wechsler D. WAIS-III: administration and scoring manual. Psychol Corp. 1997.

42. Borg GA. Psychophysical bases of perceived exertion. Med Sci Sports Exerc. 1982;14:377-81.

43. Okamoto M, Dan H, Sakamoto K, Takeo K, Shimizu K, Kohno S, Oda I, Isobe S, Suzuki T, Kohyama K, Dan I. Three-dimensional probabilistic anatomical cranio-cerebral correlation via the international 10-20 system oriented for transcranial functional brain mapping. Neuroimage. 2004;21:99-111.

44. Maidan I, Bernad-Elazari H, Gazit E, Brozgol M, Giladi N, Mirelman A, Hausdorff $J M$. Increased activation of the frontal lobe is associated with freezing of gait in patients with Parkinson's disease: an fNIRS study. Mov Disord. 2015;28:S324.

45. Sakatani K, Yamashita D, Yamanaka T, Oda M, Yamashita Y, Hoshino T, Fujiwara N, Murata Y, Katayama Y. Changes of cerebral blood oxygenation and optical pathlength during activation and deactivation in the prefrontal cortex measured by time-resolved near infrared spectroscopy. Life Sci. 2006;78:2734-41.

46. Duncan A, Meek JH, Clemence M, Elwell CE, Fallon P, Tyszczuk L, Cope M, Delpy DT. Measurement of cranial optical path length as a function of age using phase resolved near infrared spectroscopy. Pediatr Res. 1996;39:889-94.

47. Scholkmann F, Spichtig S, Muehlemann T, Wolf M. How to detect and reduce movement artifacts in near-infrared imaging using moving standard deviation and spline interpolation. Physiol Meas. 2010;31:649-62.

48. Bilney B, Morris M, Webster K. Concurrent related validity of the GAITRite walkway system for quantification of the spatial and temporal parameters of gait. Gait Posture. 2003;17:68-74.

49. Chien SL, Lin SZ, Liang CC, Soong YS, Lin SH, Hsin YL, Lee CW, Chen SY. The efficacy of quantitative gait analysis by the GAITRite system in evaluation of parkinsonian bradykinesia. Parkinsonism Relat Disord. 2006;12:438-42.

50. Menz HB, Latt MD, Tiedemann A, Mun San Kwan M, Lord SR. Reliability of the GAITRite walkway system for the quantification of temporo-spatial parameters of gait in young and older people. Gait Posture. 2004;20:20-5.

51. Lord S, Rochester L, Baker K, Nieuwboer A. Concurrent validity of accelerometry to measure gait in Parkinsons Disease. Gait Posture. 2008;27:357-9.

52. Faul F, Erdfelder E, Lang AG, Buchner A. G*Power 3: a flexible statistical power analysis program for the social, behavioral, and biomedical sciences. Behav Res Methods. 2007;39:175-91.

53. Cohen J. Statistical power analysis for the behavioral sciences. 3rd ed. New York: Academic Press; 1988

54. Holtzer R, Verghese J, Allali G, Izzetoglu M, Wang C, Mahoney JR. Neurological gait abnormalities moderate the functional brain signature of the posture first hypothesis. Brain Topogr. 2016;29:334-43.

55. Haeussinger FB, Heinzel S, Hahn T, Schecklmann M, Ehlis AC, Fallgatter AJ. Simulation of near-infrared light absorption considering individual head and prefrontal cortex anatomy: implications for optical neuroimaging. PLOS ONE. 2011;6, e26377.

56. Atsumori $H$, Kiguchi $M$, Obata $A$, Sato $H$, Katura $T$, Funane $T$, Maki A. Development of wearable optical topography system for mapping the prefrontal cortex activation. Rev Sci Instrum. 2009;80:043704.

57. Piper SK, Krueger A, Koch SP, Mehnert J, Habermehl C, Steinbrink J, Obrig H, Schmitz $\mathrm{CH}$. A wearable multi-channel fNIRS system for brain imaging in freely moving subjects. Neuroimage. 2014;85(Pt 1):64-71.

58. Heinzel S, Haeussinger FB, Hahn T, Ehlis AC, Plichta MM, Fallgatter AJ. Variability of (functional) hemodynamics as measured with simultaneous fNIRS and fMRI during intertemporal choice. Neuroimage. 2013;71:125-34.

59. Gagnon L, Cooper RJ, Yucel MA, Perdue KL, Greve DN, Boas DA. Short separation channel location impacts the performance of short channel regression in NIRS. Neuroimage. 2012;59:2518-28.

60. Kirilina E, Jelzow A, Heine A, Niessing M, Wabnitz H, Bruhl R, Ittermann B, Jacobs AM, Tachtsidis I. The physiological origin of task-evoked systemic artefacts in functional near infrared spectroscopy. Neuroimage. 2012;61:70-81.
61. Takahashi T, Takikawa Y, Kawagoe R, Shibuya S, Iwano T, Kitazawa S. Influence of skin blood flow on near-infrared spectroscopy signals measured on the forehead during a verbal fluency task. Neuroimage. 2011;57:991-1002.

62. Sato $H$, Yahata N, Funane $T$, Takizawa R, Katura T, Atsumori H, Nishimura $Y$, Kinoshita A, Kiguchi M, Koizumi H, et al. A NIRS-fMRI investigation of prefrontal cortex activity during a working memory task. Neuroimage. 2013;83:158-73.

63. Cui X, Bray S, Bryant DM, Glover GH, Reiss AL. A quantitative comparison of NIRS and fMRI across multiple cognitive tasks. Neuroimage. 2011;54:2808-21.

\section{Submit your next manuscript to BioMed Central and we will help you at every step:}

- We accept pre-submission inquiries

- Our selector tool helps you to find the most relevant journal

- We provide round the clock customer support

- Convenient online submission

- Thorough peer review

- Inclusion in PubMed and all major indexing services

- Maximum visibility for your research

Submit your manuscript at www.biomedcentral.com/submit
C Biomed Central 